\title{
EFICÁCIA DO CARFENTRAZONE-ETHYL EM MISTURA COM GLYPHOSATE NO CONTROLE DE TRAPOERABA NA CULTURA DO CAFÉ
}

\author{
Luciano S. de Souza ${ }^{1}$, Alexandre Buim ${ }^{2}$, \\ José Irineu B. Pereira Filho ${ }^{3}$ e Vincenzo de Palma ${ }^{1}$
}

\author{
${ }^{\prime} E^{\circ}{ }^{\circ}$ Agro, Dr., Professor. FCA/UNIMAR. Av. Higyno Muzzi Filho, 1001. Marília, SP 17525-902 lsouza-ca@unimar.br \\ ${ }^{2}$ Graduando de Agrônomia, FCA/UNIMAR. \\ ${ }^{3}$ Engenheiro Agrônomo. Rua Tiradentes, 76. Garça, SP 17400-000 jose-irineu $\omega$,uol.com.br
}

\begin{abstract}
RESUMO
O objetivo do presente trabalho foi avaliar a eficácia do herbicida carfentrazone-ethyl em mistura com glyphosate no controle de trapoeraba (Commelina benghalensis) na cultura do café (Coffea arabica). O experimento foi conduzido na Fazenda São José, localizada no município de Garça, SP. Os tratamentos utilizados foram: carfentrazone + glyphosate (10+2500. $15+1200,20+1200,25+1200,30+1200,40+1200$ e 50+1200 g/ha), 2,4-D (2680 g/ha), glyphosate (1200 g/ha) e uma testemunha sem controle. Foi utilizado o delineamento experimental em blocos ao acaso com 3 repetições. A combinação de carfentrazone-ethyl com glyphosate foi eficiente no controle de trapoeraba a partir da dose de $75 \mathrm{~g} / \mathrm{ha}$. A máxima dose utilizada de carfentrazone-ethyl (125 g/ha) na combinação com glyphosate, provocou total controle de trapoeraba aos 7 dias após os tratamentos. O glyphosate foi mais eficaz no controle das plantas daninhas e provocou dessecação mais rápida, quando foram utilizadas doses crescentes de carfentrazone acima de $25 \mathrm{~g} / \mathrm{ha}$ (meia dose) comparado com o herbicida glyphosate aplicado isoladamente. Todos os herbicidas testados não apresentaram toxicidade nas folhas de café.
\end{abstract}

Palavras-chave: Coffea arabica, Commelina benghalensis, herbicida, dessecante, planta daninha.

\section{ABSTRACT \\ Efficacy of carfentrazone-ethyl and glyphosate mixtures on control of Commelina benghalensis in coffee crop (Coffea arabica)}

The objective of this research was to evaluate the efficacy in control of Commelina benghalensis using mixtures of carfentrazone-ethyl and glyphosate herbicides on the coffee crop. The experiment was carried out in São José Farm, GarçaSP, Brazil. The treatments were: postemergence application of carfentrazone-ethyl + glyphosate $(10+1200,15+1200,20+1200$, $25+1200,30+1200,40+1200$ and $50+1200 \mathrm{~g} / \mathrm{ha}), 2,4-\mathrm{D}(2680 \mathrm{~g} / \mathrm{ha})$, glyphosate $(1200 \mathrm{~g} / \mathrm{ha})$ and an untreated control. Experimental design was a randomized complete block with three replications. The carfentrazone-ethyl mixture with glyphosate herbicide was efficient in the control of Commelina benghalensis starting with doses greater than or equal to $30 \mathrm{~g} / \mathrm{ha}$. The maximum dose of carfentrazone-ethyl ( $50 \mathrm{~g} / \mathrm{ha})$ mixture and glyphosate herbicides were fully efficient in control of the weed, up to 15 days after application on the coffee crop. The glyphosate herbicide control on the weed species was more efficient, presenting a faster dessication period, when used with increasing doses of carfentrazone herbicide above $25 \mathrm{~g} / \mathrm{ha}$, when 
compared with glyphosate alone. The evaluated herbicides and mixtures did not present toxicity to the coffee leaves.

Key words: Purslane, herbicide, desicant, weeds.

\section{INTRODUÇÃO}

Estudos realizados com o carfentrazone-ethyl têm demonstrado que este herbicida controla eficazmente plantas daninhas de folhas largas (Shires et al., 1997) como a trapoeraba (Commelina communis) (Lee et al., 1997), corda de viola (Ipomoea hederacea) (Dayan et al., 1997) e fedegoso (Chenopodium album ) (Durgan et al., 1997) em varias culturas perenes e anuais.

O carfentrazone-ethyl pertence ao grupo químico da aril triazolinona e o modo de ação consiste na inibição da formação da enzima protoporfirinogênio oxidase (PPO) responsável pela síntese da clorofila (Dayan et al., 1997). Quando aplicado nas folhas o carfentrazone é rapidamente absorvido, causando rápida dessecação foliar e por isso é pouco translocado no floema (Dayan et al., 1997).

Estudos realizados em várias culturas para a avaliação de eficácia, absorção e segurança de novos herbicidas, como o carfentrazone, são de extrema importância para o desenvolvimento de programas de controle de plantas daninhas e programas de rotação de culturas (Thompson \& Nissen, 2000).

Dentre os diferentes problemas encontrados pelo cafeicultor destaca-se o controle de plantas daninhas, que pode reduzir em até $36 \%$ a produção e afetar a qualidade da bebida (Foloni, 2000). Um dos herbicidas mais utilizados na cultura de café é o glyphosate que apresenta amplo espectro de controle de plantas daninhas, principalmente gramineas. Entretanto, esse herbicida apresenta baixo controle de trapoeraba.

O objetivo do presente trabalho foi avaliar a eficácia do herbicida carfentrazone-ethyl, em mistura com glyphosate, no controle de trapoeraba e espécies de plantas daninhas de ocorrência na cultura do café, na região de Marília-SP.

\section{MATERIAL E MÉTODOS}

O experimento foi conduzido na Fazenda São José, localizada no município de Garça-SP. O solo da área experimental é classificado como Podzólico Vermelho-Amarelo, transição abrupta. Segundo a classificação climática de Koeppen, o clima predominante na região é do tipo $\boldsymbol{C} \boldsymbol{w} \boldsymbol{a}$. É caracterizado pelo clima tropical de altitude, com inverno seco e verão úmido (Lombardi Neto \& Drugowich, 1994).

Utilizou-se a variedade de café Mundo Novo, plantado no ano de 1970 e recepado em 1998. O espaçamento foi de $2,5 \mathrm{~m}$ na linha de plantio e $3,8 \mathrm{~m}$ nas entrelinhas. Os tratamentos aplicados no modo pós-mato/pós-plantio na cul- tura do café foram: carfentrazone-ethyl+glyphosate $(10+1200$, $15+1200,20+1200,25+1200,30+1200,40+1200$ e $50+1200$ $\mathrm{g} / \mathrm{ha})$, 2,4-D (2680 g/ha), glyphosate (1200 g/ha) e uma testemunha sem controle. Foi utilizado o delineamento experimental em blocos ao acaso, com 3 repetições. Cada parcela foi composta de 2 linhas de plantio com $6 \mathrm{~m}$ de comprimento, perfazendo um total de $22,8 \mathrm{~m}^{2}$.

Os herbicidas utilizados foram formulações comerciais de glyphosate (480 g/l i.a.), 2,4-D (670 g/l i.a.) e carfentrazone-ethyl $400 \mathrm{CE}$ (400 g/l i.a.). A aplicação dos herbicidas foi realizada em 9 de março de 2000, em pósemergência total das plantas daninhas. $\mathrm{O}$ equipamento utilizado na aplicação dos tratamentos químicos foi um pulverizador costal pressurizado a $\mathrm{CO}_{2}$, equipado com barra de aplicação munido de pontas (bicos) Teejet $110.02 \mathrm{XR}$, calibrados para $2,8 \mathrm{~kg} / \mathrm{cm}^{2}$ de pressão, consumindo $150 \mathrm{l} / \mathrm{ha}$ de calda. As misturas de carfentrazone-ethyl com glyphosate foram aplicadas com óleo mineral, na concentração de $0,5 \% \mathrm{v} / \mathrm{v}$.

Por ocasião da aplicação dos herbicidas a temperatura e umidade relativa do ar foram em média de $30^{\circ} \mathrm{C}$ e $85 \%$, respectivamente. $O$ vento apresentava-se em forma de rajada e oscilava entre 1,0 e $1,2 \mathrm{~m} / \mathrm{s}$.

$\mathrm{Na}$ época da aplicação a comunidade infestante estava composta de trapoeraba (Commelina benghalensis), que compunha $50 \%$ da flora no estádio de 6 a 7 folhas, além de capim-colchão (Digitaria horizontalis) e capim-carrapicho (Cenchrus echinatus), ambas no estádio de 5 a 8 folhas, capim-braquiária (Brachiaria decumbens) no estádio de 3 a 6 folhas, picão-preto (Bidens pilosa) e poaia-branca (Richardia brasiliensis), ambas no estádio de 2 a 4 folhas, cada uma delas representando $10 \%$ da comunidade infestante. Todas estas espécies são plantas de ocorrência na cultura do café na região de Maríla e Garça, no Estado de São Paulo.

O controle das plantas daninhas foi avaliado visualmente aos 7, 15, 21 e 30 dias após a aplicação dos tratamentos (DAT), adotando-se a escala percentual de notas, sendo zero correspondente a nenhum controle e cem equivalente a controle total das plantas daninhas. Na mesma época foi avaliada a toxicidade sobre as plantas de café, adotando-se também, a escala percentual descrita acima. Os resultados foram submetidos à análise de variância pelo teste $\mathrm{F}$ e as médias comparadas pelo teste de Duncan a $5 \%$ de probabilidade.

\section{RESULTADOS E DISCUSSÃO}

Os tratamentos com a mistura de carfentrazone-ethyl com glyphosate provocaram efeitos crescentes de controle da trapoeraba com o aumento da dose de carfentrazone-ethyl, verificada para todas as avaliações (Tabelas 1 e 2). Obser- 
vou-se que a partir dos 15 dias após o tratamento (DAT) os efeitos de controle foram mais lentos nas menores doses de carfentrazone. Os efeitos mais drásticos e rápidos foram observados nas doses de carfentrazone acima de $30 \mathrm{~g} / \mathrm{ha}$, quando a percentagem de controle aumentar para $100 \%$ na maior dose, a partir dos 21 DAT.

Estes resultados de carfentrazone-ethyl + glyphosate foram evidenciados por Lee et al. (1997), que avaliaram o carfentrazone-ethyl isolado e em combinação com glyphosate ou glufosinate no controle de trapoeraba (Commelina communis) e várias plantas daninhas, na cultura da pêra. Os resultados de Lee et al. (1997) mostraram ainda que a combinação de carfentrazone com glyphosate foi melhor que com glufosinate no controle das plantas daninhas analisadas, principalmente de capim-arroz (Echinochloa crusgalli).

O herbicida glyphosate, aplicado isoladamente, não

Tabela 1. Percentagens médias de controle de Commelina benghalensis aos 7, 15,21 e 30 dias após a aplicação dos tratamentos (DAT), na cultura do café. Marília, SP. 2000.

\begin{tabular}{|c|c|c|c|c|c|}
\hline \multirow{3}{*}{ Tratamento } & \multirow{3}{*}{$\begin{array}{c}\text { Dose } \\
\text { (g/ha) }\end{array}$} & \multicolumn{4}{|c|}{ Controle $(\%)$} \\
\hline & & \multicolumn{4}{|c|}{ DAT } \\
\hline & & 7 & 15 & 21 & 30 \\
\hline 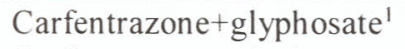 & $50+1200$ & $94 a^{2}$ & $96 \mathrm{a}$ & $100 \mathrm{a}$ & $100,0 \mathrm{a}$ \\
\hline Carfentrazone+glyphosate & $40+1200$ & 88 a & $90 \mathrm{~b}$ & $98 \mathrm{a}$ & 98 a \\
\hline Carfentrazone+glyphosate & $30+1200$ & $62 b$ & $73 \mathrm{c}$ & $85 \mathrm{~b}$ & $85 \mathrm{~b}$ \\
\hline Carfentrazone+glyphosate & $25+1200$ & $43 \mathrm{c}$ & $53 \mathrm{~d}$ & $65 c$ & $67 c$ \\
\hline Carfentrazone+glyphosate & $20+1200$ & $38 \mathrm{~cd}$ & $47 \mathrm{~d}$ & $65 \mathrm{c}$ & $67 \mathrm{c}$ \\
\hline Carfentrazone+glyphosate & $15+1200$ & $28 \mathrm{~d}$ & $30 \mathrm{e}$ & $52 \mathrm{~d}$ & $52 \mathrm{~d}$ \\
\hline Carfentrazone+glyphosate & $10+1200$ & $15 \mathrm{e}$ & $20 \mathrm{e}$ & $35 \mathrm{e}$ & 37 e \\
\hline $2,4, \mathrm{D}$ & 2680 & $38 \mathrm{~cd}$ & $45 \mathrm{~d}$ & $47 \mathrm{de}$ & $85 \mathrm{~b}$ \\
\hline Glyphosate & 1200 & 12 ef & $20 \mathrm{e}$ & $35 \mathrm{e}$ & $40 \mathrm{e}$ \\
\hline Testemunha & - & $0 \mathrm{f}$ & $0 \mathrm{f}$ & $0 \mathrm{f}$ & $0 \mathrm{f}$ \\
\hline F tratamentos & - & $60,38^{* *}$ & $51,17^{* *}$ & $66,02^{* *}$ & $78,23^{* x}$ \\
\hline C.V. (\%) & - & 12,0 & 14,5 & 16,1 & 14,9 \\
\hline
\end{tabular}

'Às misturas de carfentrazone com glyphosate foi adicionado óleo mineral a $0.5 \%$ (v.v.).

${ }^{2}$ Médias seguidas por uma mesma letra na coluna, não diferem entre si pelo teste de Duncan a $5 \%$ de probabilidade.

** Significativo ao nível de $5 \%$ de probabilidade.

Tabela 2. Percentagens médias de controle do total de várias espécies de plantas daninhas (capim-colchão, capim-braquiária. capim-carrapicho, picão-preto, e poaia-branca), aos 7, 15,21 e 30 dias após a aplicação dos tratamentos (DAT), na cultura do café. Marília, SP. 2000.

\begin{tabular}{|c|c|c|c|c|c|}
\hline \multirow{3}{*}{ Tratamentos } & \multirow{3}{*}{$\begin{array}{c}\text { Dose } \\
\text { (g/ha) }\end{array}$} & \multicolumn{4}{|c|}{ Controle $(\%)$} \\
\hline & & \multicolumn{4}{|c|}{ DAT } \\
\hline & & 7 & 15 & 21 & 30 \\
\hline Carfentrazone+glyphosate & $50+1200$ & $83 \mathrm{a}^{2}$ & $95 \mathrm{a}$ & $98 \mathrm{a}$ & $99 \mathrm{a}$ \\
\hline Carfentrazone+glyphosate & $40+1200$ & $75 \mathrm{a}$ & $85 \mathrm{~b}$ & $94 \mathrm{ab}$ & $96 \mathrm{ab}$ \\
\hline Carfentrazone+glyphosate & $30+1200$ & $62 \mathrm{~b}$ & $83 \mathrm{~b}$ & $93 \mathrm{~b}$ & $96 \mathrm{ab}$ \\
\hline Carfentrazone+glyphosate & $25+1200$ & $55 \mathrm{~b}$ & $78 \mathrm{~b}$ & $90 \mathrm{bc}$ & $93 \mathrm{~b}$ \\
\hline Carfentrazone+glyphosate & $20+1200$ & $55 \mathrm{~b}$ & $77 \mathrm{~b}$ & $88 \mathrm{~cd}$ & $93 \mathrm{~b}$ \\
\hline Carfentrazone+glyphosate & $15+1200$ & $53 \mathrm{~b}$ & $67 \mathrm{c}$ & 85 de & $92 \mathrm{~b}$ \\
\hline Carfentrazone+glyphosate & $10+1200$ & $52 \mathrm{~b}$ & $67 \mathrm{c}$ & $83 \mathrm{e}$ & $92 \mathrm{~b}$ \\
\hline $2,4-\mathrm{D}$ & 1680 & $12 \mathrm{c}$ & $15 \mathrm{~d}$ & $18 \mathrm{f}$ & $20 \mathrm{c}$ \\
\hline Glyphosate & 1200 & $53 \mathrm{~b}$ & $77 \mathrm{~b}$ & $92 \mathrm{bc}$ & $95 \mathrm{ab}$ \\
\hline Testemunha & - & $0 \mathrm{~d}$ & $0 \mathrm{e}$ & $0 \mathrm{~g}$ & $0 \mathrm{~d}$ \\
\hline F tratamentos & & $66,36^{* *}$ & $123,44^{* *}$ & $512,89 * *$ & $579,0^{* *}$ \\
\hline C.V. (\%) & & 10,9 & 13,7 & 10,4 & 11,6 \\
\hline
\end{tabular}

${ }^{1}$ As misturas de carfentrazone com glyphosate foi adicionado óleo mineral a $0.5 \%$ (v.v.).

${ }^{2}$ Médias seguidas por uma mesma letra na coluna, nào diferem entre si pelo teste de Duncan a $5 \%$ de probabilidade.

** Significativo ao nível de $5 \%$ de probabilidade 
proporcionou controle da trapoeraba, sendo que a melhor percentagem de controle, aos 30 DAT (40\%), foi correspondente à menor dose utilizada de carfentrazone-ethyl $(25 \mathrm{~g} / \mathrm{ha})$.

O controle das outras espécies de plantas daninhas (capim-colchão, capim-braquiária, capim-carrapicho, picãopreto, e poaia-branca), foi mais eficaz, com menor tempo de dessecação, quando se utilizaram doses crescentes de carfentrazone acima de $20 \mathrm{~g} / \mathrm{ha}$ (meia dose).

Resultados semelhantes foram encontrados por Foloni (2000). Na menor dose, o carfentrazone propiciou melhor desempenho do glyphosate, quer no aspecto de velocidade de controle, quanto no controle das diferentes espécies presentes, mostrando a possibilidade real de tornar-se um parceiro deste produto no manejo de plantas daninhas. Enquanto isso nos tratamentos com o herbicida 2,4-D as percentagens de controle reduziram-se para a faixa de $20 \%$, tornando-se insuficiente. devido ao não controle das plantas daninhas de folhas estreitas.

Todos os herbicidas testados não apresentaram sintomas de toxicidade nas folhas de café.

\section{CONCLUSÕES}

A combinação de carfentrazone-ethyl com gliphosate foi eficiente no controle de trapoeraba (Commelina benghalensis), na cultura do café, a partir da dose de $30 \mathrm{~g} /$ ha.

A máxima dose utilizada de carfentrazone-ethyl (50 $\mathrm{g} / \mathrm{ha}$ ) em combinação com glyphosate, provocou total controle de trapoeraba a partir dos 21 DAT.

Todos os herbicidas testados não apresentaram sintomas de toxicidade nas folhas de café.

\section{LITERATURA CITADA}

DAYAN, F.E.; DUKE, S.O.; WEETE, J.D.; HANCOCK, H.G. Selectivivity and mode of action of carfentrazone-ethyyl, a novel phenyl triazolinone hercicide. Pesticide Science, v.51, n.1, p.65-73, 1997.

DURGAN, B.R.; YENISH, J.P.; DAML, R.J.; MILLER, D.W. Broadleaf weed control in hard red spring wheat (Triticum aestivum) with F8426. Weed Technology, v.11, n.3, p.489-495, 1997.

FOLONI, L.L. Eficiência e seletividade da mistura de glifosate + carfentrazone-ethyl, em pós-emergência, na cultura de café novo. In: CONGRESSO BRASILEIRO DE PESQUISAS CAFEEIRAS, 26, 2000, Marília. Resumos... Marília: MA/PROCAFÉ, p. 197, 2000.

LEE, H.J.; HAN, S.U.; GUH, J.O.; KIM, M.H. Evaluation of carfentrazone-ethyl alone and in combination with glyphosate or glufosinate for weed control in orchards. Korean Journal of Weed Science, v.17, n. 3, p.256261, 1997.

LOMBARDI NETO, F.; DRUGOWICH. M.I. Manual técnico de manejo e conservação de solo e água. Vol.2. Campinas: CATI, 1994. 168 p.

SHIRES, S.W.; BOURDOUXHE, L.A.; CROSSMAN, A.R.; GENOT, B.; LAFFRANQUE, J.P.; LEBRANC, J. Carfentrazone-ethyl: a new herbicide for the rapid control of key. In: BRIGHTON CROP PROTECTION CONFERENCE: Weeds. Proceedings... Brighton, v. 1 . p.117-122, 1997.

THOMPSON, W.M.: NISSEN, S.J. Absorption and fate of carfentrazone-ethyl in Zea mays. Glycine max, and Abutilon theophrasti. Weed Science, v.48, n.1, p.1519, 2000. 